\title{
The role of visual acuity and segmentation cues in compound word identification
}

\author{
Jukka Hyönä* \\ Department of Psychology, University of Turku, Turku, Finland
}

\section{Edited by:}

Jon Andoni Dunabeitia, Basque

Center on Cognition, Brain and

Language, Spain

Reviewed by:

Alexander Pollatsek, University of

Massachusetts Amherst, USA

Barbara Juhasz, Wesleyan University,

USA

Jukka Hyönä, Department of

Psychology, University of Turku,

Fl-20014 Turku, Finland.

e-mail: hyona@utu.fi
${ }^{*}$ Correspondence:

Studies are reviewed that demonstrate how the identification of compound words during reading is constrained by the foveal area of the eye. When compound words are short, their letters can be identified during a single fixation, leading to the whole-word route dominating word recognition from early on. Hence, marking morpheme boundaries visually by means of hyphens slows down the processing of short words by encouraging morphological decomposition when holistic processing is a feasible option. In contrast, the decomposition route dominates the early stages of identifying long compound words. Thus, visual marking of morpheme boundaries facilitates processing of long compound words, unless the initial fixation made on the word lands very close to the morpheme boundary. The reviewed pattern of results is explained by the visual acuity principle (Bertram and Hyönä, 2003) and the dual-route framework of morphological processing.

Keywords: word recognition, reading, compound words, eye movements, morphological processing, hyphenation, fovea

Research into the identification of compound words shows that word length is a central factor that should be taken into account when determining what happens in the first instances of the reading process. In the study of printed word recognition, it is tempting to restrict attention to words of a particular length. So far, the emphasis has been on the identification of short words (typically four to six letters). This choice may be motivated for many analytic languages, in which average word length tends to be quite short. However, in agglutinative languages where by default words comprise multiple morphemes, words tend to be significantly longer than in analytic languages. Finnish is a good example of a highly inflecting agglutinative language. For example, the multimorphemic word autoissammekin would be translated in an analytic language using multiple words: also in our cars. Thus, in order to gain a more complete picture of printed word recognition across structurally different languages, more emphasis should be placed on the investigation of recognizing long, multimorphemic words.

In the present review, the focus is on word compounding, which is a typical feature in agglutinative languages (e.g., Finnish), but it can also be found in more analytic languages (e.g., Dutch, German, Swedish). I review results regarding the identification of compound words during sentence reading. The reviewed studies have examined effects of two consequences of word compounding: (1) Compound words containing multiple morphemes tend to be rather long; (2) by not being marked by visually salient cues, such as spaces between morphemes, within-word morphemic units may become obscured, which may impede recognition. I particularly focus on studies examining effects of word length and salience of morpheme boundaries on the identification of compound words during reading. Thus, the present review does not cover all eye movement studies conducted on compound word reading (for a review of studies not fully covered here, see Pollatsek and Hyönä, 2006).
It is well documented that word length has a robust effect on word recognition. During reading, long words take more time to be recognized than short words (e.g., Just and Carpenter, 1980; Hyönä and Olson, 1995; Calvo and Meseguer, 2002; Kliegl et al., 2004; Juhasz et al., 2008). This is largely, but not entirely (McDonald, 2006; Hautala et al., 2011), due to long words being much more likely to require a second eye fixation on the word for successful recognition. A refixation is needed due to visual acuity limitations of the human eye.

Visual acuity drops dramatically as a function of the distance from the current fixation center. Vision is sharpest around the fovea, which spans about $2^{\circ}$ of visual angle around the center of the fixation point. For adult readers the letter identity span (the region within which letter identities can be recognized) is no more than nine letters to the right of fixation (Häikiö et al., 2009). The span is also attentionally modulated so that it is greater toward right than left when reading text from left to right (Rayner et al., 1980, 1982); the leftward span is limited to the beginning of the currently fixated word. As the initial fixation tends to land somewhat left of the word center (Rayner, 1979), the letter (and word) identification span for adult readers is no more than 12 letters (asymmetric to the right). It should be noted, however, that the most typical fixation strategy for reading 12-letter compound words is nevertheless a two-fixation strategy (e.g., Hyönä and Pollatsek, 1998).

Long words do not only differ from short words in that they have more letters. As was briefly noted above, increased length also makes it more likely that words contain multiple morphemes. The fact that within-word morphemic units are not visually separable from each other results in the structure of multimorphemic words not being visually transparent, which in turn may lead to processing difficulties. Word length may exacerbate the impact of the structural opacity. With increased length, words are more likely to contain multiple morphemes. Moreover, decomposing 
morphemes from each other may become more difficult the further away morphemes and morpheme boundaries are from the current fixation point. Thus, the probability of refixating a word as a function of morphological complexity is likely to increase even when all letters of the word are within the limits of the letter identity span.

In summary, longer word length increases chances of refixation in two ways: a higher number of letters reduces the visual acuity for words as a whole, and longer words are more likely to be made up of several morphemic units, which complicates interpretation. In the next two sections, I will discuss experiments investigating both issues. Finally, in the last section I will argue that the results of these studies strongly suggest that both number of letters and structural opacity affect early processing during reading by means of the visual acuity principle. I will also show how this explanation fits within the dual-route framework of morphological processing.

\section{THE ROLE OF WORD LENGTH IN THE IDENTIFICATION OF COMPOUND WORDS}

This section discusses the first main topic of this review: the effect of word length on the identification of morphologically complex words. As noted above, an increase in word length is accompanied by an increase in the probability of the reader not being able to read a word with a single fixation; instead, a refixation is programmed to the word region falling outside the letter identity span. In other words, when parallel processing of all letters of the word is rendered impossible due to visual acuity limitations, longer words are recognized sequentially. During the second stage (i.e., during the refixation), letters initially falling outside the letter identity span are subsequently identified. It should be noted, however, that during the initial stage the non-identified letters are pre-processed, leading to a subsequent processing benefit (called preview benefit) during their foveal processing (for recent reviews of parafoveal processing during reading, see Hyönä, 2011; Schotter et al., 2012).

But how does word length influence the identification of multimorphemic words? This question was investigated by Bertram and Hyönä (2003) with two-constituent Finnish compound words that were either relatively short (an average of 7.6 letters) or long (an average of 12.8 letters). The target words were embedded in sentences; participants' eye movements were tracked while they were reading these sentences for comprehension. In Experiment 1, the frequency of the first-constituent (as a separate word) was manipulated for both short and long compound words; in Experiment 2 the same was done for the whole-word frequency. According to the logic adopted from Taft and Forster's (1976) seminal work, an early effect of first-constituent frequency would suggest that the compound word is decomposed for its recognition (lexical access is initiated by the recognition of the first-constituent, followed by the recognition of the second constituent and the whole-word). On the other hand, parallel processing of the two components is implicated by an early effect of whole-word frequency and by the absence of an early effect of first-constituent frequency.

An attractive feature of the eye-tracking technique is that it can be used to tap into the time course of processing, particularly when word processing is distributed across multiple fixations. Hence, the duration of the first-fixation can be used to index early processes, while durations of subsequent fixations reflect processing done at later stages. Despite being an aggregate measure, gaze duration (i.e., the summed duration of fixations landing on the word during its first-pass reading) is typically also used as an index of relatively late processing. This is due to gaze duration strongly reflecting the probability of refixating a word.

In Experiment 1, an early effect of first-constituent frequency, as indexed by first-fixation duration, was obtained for long compound words but not for short compound words; the latter only revealed a marginal effect in later processing indexed by gaze duration. In contrast, Experiment 2 revealed are liable early effect of whole-word frequency for short compound words but only a small and statistically marginal effect ( $4 \mathrm{~ms}$ ) for long compound words; both types of words showed a whole-word frequency effect in later processing, as indexed by gaze duration. Thus, the pattern of data suggests that for short compound words the whole-word representation becomes active soon after the word is foveated. On the other hand, with long compound words the first-constituent is more strongly activated during the initial processing stage than the whole-word representation. It should be noted, however, that even though the whole-word representation receives early activation for short compound words, short compound words are not fully identified during the initial fixation, but often (roughly about half of the trials in the Bertram and Hyönä study) a refixation is needed to complete the lexical access.

To account for the observed pattern of results, Bertram and Hyönä (2003) put forth the visual acuity principle. According to this principle, word processing is initiated with whatever information is readily available in the foveal vision. When all (or a sufficient number of) letters of the word are within foveal reach, the whole-word representation becomes readily available early on. On the other hand, when only the initial morpheme is foveally available, as is the case with long compound words (longer than about 12 letters), word recognition is initiated by first accessing the initial constituent, followed by the second constituent and the whole-word. The claim that there is a strong sequential component in recognizing long compound words is further supported by the finding that the earliest point in time when the frequency of second constituent exerts an effect is when a second fixation is made on the word (Pollatsek et al., 2000). Note, however, that Inhoff et al. (2008) reported evidence indicating that the frequency of the second lexeme already exerts an effect on the first-fixation duration. This effect was obtained for the so-called tailed compound words, for which the second lexeme was the meaning-defining lexeme. However, the effect was not significant in the item analysis. Considering that their compound words varied in length between 8 and 11 letters (average length 9.1 letters), it is possible that the early second lexeme frequency effect was produced by the shorter compounds. If so, it would be evidence for compound word lexemes playing an active role early on during the identification of short compounds - a claim inconsistent with the visual acuity principle of Bertram and Hyönä (2003) and with the data of Pollatsek et al. (2000).

With regards to existing models of morphological processing, the data of Bertram and Hyönä (2003) are consistent with parallel dual-route morphological models (e.g., Schreuder and Baayen, 1995; Pollatsek et al., 2000). These models assume two routes to be in operation in tandem: the decomposition route and the full-form 
route. Lexical access via the decomposition route takes place via the constituents, while the full-form route attempts access by finding a match between the visual input and a stored whole-word representation. To account for the above-summarized results, this framework needs to be complemented with the visual acuity principle. A head-start is won by the route favored by visual acuity. In other words, when only the first-constituent is fully available in the fovea, the decomposition route achieves a head-start; in contrast, the full-form route is either initially favored when the whole-word is within foveal reach or it quickly overtakes the initially favored decomposition route. The later effect of whole-word frequency for long compounds and the later effect of first-constituent frequency for short compounds observed by Bertram and Hyönä (2003) may in this framework be taken as reflecting the later activation of the slower route.

Does the full-form route provide access only to the lexical representation of the compound word or is access to meaning simultaneously also achieved? My present view is that with existing (lexicalized) compound words lexical access is very quickly followed by the activation of word meaning. My view is based on two eye-tracking experiments (Pollatsek and Hyönä, 2005; Frisson et al., 2008) that did not find evidence for disruption in processing when compound words were semantically opaque, in comparison to semantically transparent compounds (see, however, Juhasz, 2007). On the other hand, with novel compound words for which no mental representation exists, a meaning computation stage is quite naturally required (Pollatsek et al., 2011).

More recently, Fiorentino and Poeppel (2007) have studied the time course of compound word processing by registering brain activation via MEG (magnetoencephalography) when participants made lexical decisions to frequency- and length-matched compound and monomorphemic words in English. Their stimuli were comparable in length (an average of 7.8 letters) to the short compounds of Bertram and Hyönä (2003). All words were infrequent; however, the compound word constituents were all frequent (as separate words). There were two main findings: (a) lexical decision time was shorter for compound words than for frequency-matched monomorphemic words; (b) the MEG component presumed to index lexical access (M350) peaked earlier for compound than for monomorphemic words. These data were taken to suggest that compound words are always recognized via the decomposition route. This conclusion contrasts with the argument made by Bertram and Hyönä (2003). To recap, they posit that the fullform route has a head-start in processing compound words which are sufficiently short to fit in the foveal area of the eye.

There are two plausible explanations to account for the apparent discrepancy between the two set of results. First, Fiorentino and Poeppel (2007) presented their stimuli in a very large font; on average the words extended horizontally $6.4^{\circ}$ of visual angle, which means that the words did not fit in the foveal area. Thus, the visual acuity principle predicts here that the decomposition route is initially favored - a claim consistent with their data. Second, the processing disadvantage for monomorphemic over compound words may be strengthened by the fact that a subset of the monomorphemic words was probably quite unknown to the participants. This possibility is hinted at by the fact that participants made $20 \%$ decision errors with the lowest-frequency monomorphemic words. At any rate, further studies are needed to solve this discrepancy. An attractive possibility would be to combine eye-tracking with MEG recordings to examine whether the two methods provide converging evidence concerning the timing of different frequency effects. It would also serve as a methodological cross-validation.

Vergara-Martínez et al. (2009) conducted an ERP study in Basque, where they independently varied the frequency of the first and second constituent in two-constituent compound words. The target words were embedded in sentence context, and participants were asked to read the sentence for comprehension. Importantly for the present discussion, the length of the target words varied from 6 to 12 letters (average length 9.25 letters). Their spatial extent in terms of visual angle is not provided in the paper. However, Duñabeitia (personal communication) informed me that their standard procedure was to use Courier New font where one character subtended horizontally $0.41 \mathrm{~cm}$. With their viewing distance of $80 \mathrm{~cm}$, a 6 -letter word subtended $1.76^{\circ}$ and 12 -letter word $3.52^{\circ}$ of visual angle, respectively. Thus, their shorter words fitted in the foveal region, while their longest words did not.

Vergara-Martínez et al. (2009) obtained an earlier electrophysical response for the first-constituent frequency manipulation than for the second constituent frequency manipulation. Moreover, the nature and the scalp distribution were different. The early negativity effect in ERPs was greater for high- than low-frequency firstconstituent compound words, whereas the amplitude of the later negativity effect was larger for low- than high-frequency second constituent compound words in the right hemisphere but not in the left hemisphere. This pattern of results was interpreted within the activation-verification framework that Duñabeitia et al. (2007) proposed for the processing of Basque and Spanish compound words. According to this framework, the early effect obtained for first-constituents reflects the activation of the morphological family triggered by first-constituents, with more activation produced by high-frequency than low-frequency first-constituents. The later effect associated with second constituents in turn reflects the selection of the final lexical candidate among those triggered by the first-constituent; the frequency of the second constituent affects the speed of verification.

The compatibility of the Vergara-Martínez et al. (2009) results with the visual acuity principle of Bertram and Hyönä (2003) cannot be readily assessed because it is unknown to what extent the observed effects should be attributed to the short and longer words. On the one hand, the average number of letters making up their compound words is closer to the average length of the short compounds used by Bertram and Hyönä. From that perspective, the early negativity effect obtained for the first-constituent manipulation may be tentatively interpreted to be inconsistent with the visual acuity principle. On the other hand, the negativity effect associated with the first-constituent frequency manipulation was also observed in the later time window - a finding compatible with those of Bertram and Hyönä (they found a suggestion for a later effect of first-constituent frequency for short compounds). It is also possible that a subset of longer compounds was responsible for the early effect of first-constituent frequency obtained by VergaraMartínez et al. (2009), whereas a subset of short compounds would be responsible for the late effect. 
Perhaps the most serious challenge to the visual acuity principle is provided by Juhasz (2008), who extended the work of Bertram and Hyönä (2003) by conducting studies in English rather than in Finnish. In Experiment 1, Juhasz employed two-constituent compounds that were either long (range: 10-13 letters; average: 10.9 letters) or short (range: six to seven letters; average: 6.6 letters), and manipulated first-constituent frequency. Contrary to Bertram and Hyönä (and several other studies), she found no early effect of first-constituent frequency for long compounds, as indexed by first-fixation duration. In contrast, for short compounds there was a nearly significant first-constituent frequency effect in first-fixation duration. The pattern of results was similar in gaze duration, with a nearly significant (not significant by items) first-constituent frequency effect for short compounds but not for long compounds (in fact, there was a marginal tendency for a reversed frequency effect).

In Experiment 2, Juhasz (2008) manipulated the rated wholeword frequency (i.e., familiarity) of short and long compounds. The frequency ratings were collected using a seven-point scale, yielding an average rating of 6.7 for the high-frequency compounds and an average rating of 3.1 for the low-frequency compounds. The short and long compounds were comparable in length to those in Experiment 1. There was a significant main effect of rated-frequency in first-fixation duration, but no interaction with word length; short and long compounds displayed an effect of similar size. However, when separate analyses were conducted for first-fixations when they were the single fixations on the word and when they were the first of multiple fixations, two opposing trends were observed. For single fixation duration, the rated-frequency effect was larger for long than short compounds, whereas the duration of first-fixation followed by at least one refixation displayed an opposite pattern. Given the fact that the latter type of trials was dominant in the Bertram and Hyönä (2003) data, these results are not completely inconsistent with their data. Finally, gaze duration revealed in the Juhasz (2008) study a larger rated-frequency effect for long than short compounds.

Taken together, the two experiments of Juhasz (2008) did not find evidence in English for the view advocated by the visual acuity principle that the decomposition route would be more powerful early on during long compound word processing, while the fullform route would quickly overtake the decomposition route when processing short compounds. At present, it is not clear how the differences in the results of Juhasz (2008) and those of Bertram and Hyönä (2003) could be explained. One possibility is that they may reflect inherent language differences: due to the morphological richness and complexity of Finnish, Finnish readers may be generally more prone to use the decomposition route than the English readers.

Before concluding the first section of the present review, I briefly discuss the possibility that the finding of early firstconstituent frequency effects being somewhat more modest in English than Finnish studies may be explained by the visual acuity principle (note, however, that the study of Juhasz, 2008, speaks to the contrary). In the English studies, the length of the compound words tended to fall somewhere between the short and long compound words used by Bertram and Hyönä (2003). Juhasz et al. (2003) studied reading processes for two-constituent English compound words that were all nine letters long. They obtained a marginal 11-ms effect of first-constituent frequency in the firstfixation duration indexing early effects. Similarly, Andrews et al. (2004) employed two-constituent compounds that were on average 8.5 letters (ranging from 6 to 11 letters) long, and found a marginal 7-8 ms effect of first-constituent frequency on first-fixation duration. On the other hand, Bertram and Hyönä (2003) observed a significant 16-ms early effect (i.e., in first-fixation duration) of first-constituent frequency for 12-14-letter Finnish compound words. These data are generally in line with the visual acuity principle, suggesting that the early involvement of first-constituents is attenuated for shorter compound words.

To sum up the first section, the data reviewed above provide relatively consistent support for the view that the identification of two-constituent compound words is constrained by word length. The results of most studies (but see Juhasz, 2008), support the hypothesis that the identification process for long compound words is initiated by first recognizing the initial constituent. In contrast, full-form access can be reached without going via the access of the constituents if compound words are short (provided that the full-form is sufficiently frequent in order to become readily available).

\section{ROLE OF SEGMENTATION CUES IN IDENTIFYING COMPOUND WORDS}

The second main topic of the present review concerns the effects of segmentation cues on the speed of identifying morphologically complex words. I have argued above that the lexical access of long compound words starts with the access of the initial constituent (i.e., via the decomposition route). If this claim is true, providing visual segmentation cues that make it easier to identify the morpheme boundary should speed up the processing of long compound words because it facilitates accessing the initial component. The same pattern should not be found for the short compound words because they are processed via the holistic route.

These claims were tested by Bertram and Hyönä (submitted) in an eye-tracking study in which participants read long (on average 12.1 letters) and short (on average 7.3 letters) compound words that were either hyphenated (e.g., musiikki-ilta) or concatenated (e.g., yllätystulos = surprise result; i.e., written without a hyphen at the constituent boundary). According to the Finnish spelling regulations (on the constraints of writing compound words in English, see Kuperman and Bertram, submitted), a hyphen has to be inserted at the constituent boundary when two identical vowels span the morpheme boundary (as in musiikki-ilta $=$ music evening). Hyphens prevent possible misparses of the syllables at the boundary, and consequently that the word's morphological structure is misparsed. By explicitly marking the multimorphemic nature of words, hyphens are likely to benefit the decomposition route but should inhibit the whole-word route. If so, a hyphen at the constituent boundary would speed up the processing of long compound words but slow down the processing of short compound words. The hyphenated and non-hyphenated compounds were matched for word frequency as well as first- and second-constituent frequency. Moreover, the number of letters (not counting the hyphen) was equated separately for the two short and long compound conditions. 
Bertram and Hyönä (submitted) obtained the predicted data pattern. The presence of hyphens in long compound words significantly affected subgaze duration (the gaze duration on the first-constituent) prior to making a saccade away from the firstconstituent. Subgaze duration was $74 \mathrm{~ms}$ shorter in the hyphenated than in the concatenated condition. An effect of similar size (64 ms) was also observed in gaze duration of the whole-word. These data are in line with the view that the presence of hyphens supports the decomposition route that is presumed to prevail during the early stages of long compound word processing. In contrast, gaze duration on short compound words was significantly longer on hyphenated than concatenated words (a difference of $43 \mathrm{~ms}$ favoring concatenated short compounds). The gaze duration effect was largely due to hyphens attracting a second fixation on short compounds (typically landing on the second constituent). In other words, in short compound words a hyphen at the morpheme boundary seemed to have boosted the decomposition route in cases where holistic processing is a viable option, as claimed by the visual acuity principle. Interestingly, Häikiö et al. (2011) replicated the detrimental effect of hyphens on the processing of short compound words with elementary school children (Second, Fourth, and Sixth graders). All children, except the slowest Second grade readers, took longer to read short compounds when these were hyphenated than when they were concatenated. These findings suggest that even relatively young readers are capable of reading short compound words via the holistic route.

In addition to providing further support for the visual acuity principle, the study of Bertram and Hyönä (submitted) also demonstrated the usefulness of visually salient morpheme boundary cues (hyphens) in reading long compound words. The usefulness of hyphens was further examined by Bertram et al. (2011). In contrast to Bertram and Hyönä (submitted), they inserted hyphens at constituent boundaries despite them not being prescribed by spelling conventions. Thus, their study was a strong test of the usefulness of segmentation cues, as the hyphens were inserted illegally. Further differences with Bertram and Hyönä were that the words consisted of three constituents instead of two, and that not only Finnish stimuli were used (lentokenttätaksi = airport taxi), but also Dutch words (e.g., voetbalbond = football association). The average length of the Dutch stimuli was 14.5 letters (range 10-21 letters) and that of the Finnish stimuli 15.8 letters (range 13-24 letters). The target compound words were inserted in single sentences; native-language participants read these sentences while their eye movements were recorded. The processing of illegally hyphenated compounds was compared to that of concatenated compounds (i.e., written as required by the spelling conventions). Hyphens were inserted either at major or minor morpheme boundaries. Major boundaries demarcate the boundary between modifier and head, as in voetbalbond (football association) or zaal-voetbal (indoor football), while minor boundaries appear at morpheme boundaries of two-constituent modifiers (e.g., voet-balbond = foot-ball association) or head (e.g., zaalvoet-ball $=$ indoor foot-ball). These two different word structures are called left-branching and right-branching, respectively. It was expected that hyphens would benefit processing when placed at major boundaries, whereas placing them at minor boundaries might lead to initially misparsing morphological structures.
For both Dutch and Finnish, Bertram et al. (2011) found a decrement in overall processing time (indexed by gaze duration on the whole-word) due to the insertion of hyphens at minor boundaries. The two sets of results differed from each other in that majorboundary hyphens speeded up gaze durations in Finnish, whereas in Dutch this condition did not differ from the concatenated words (i.e., legal spelling). More detailed analyses demonstrated early facilitation in processing hyphenated three-constituent Dutch compound words, as revealed by shorter subgaze durations on the left component (consisting of either one or two constituents, depending on branching) separated by a hyphen from the right component. In other words, subgaze duration on the modifier was shorter for illegally hyphenated compounds than for legally concatenated compounds. However, the early processing benefit was offset by a later processing cost associated with illegal hyphenation. Subgaze on the right component was significantly longer in the hyphenated than in the concatenated condition. The pattern was similar in Finnish for early processing. On the other hand, the later slowing down in processing the right component was not apparent in Finnish for the left-branching compounds (two-constituent modifier + one-constituent head) but was so for the right-branching compounds. In sum, both experiments of Bertram et al. (2011) demonstrate an early processing benefit due to hyphenation, presumably reflecting facilitation in morphological segmentation and in parsing the morphological structure (i.e., assigning the modifier-head relation) of three-constituent compound words. The later processing cost due to hyphenation is likely to reflect readers' response to illegal spelling. It is noteworthy, however, that in the course of the experiment Finnish readers became used to illegal hyphenation, to the extent that toward the end of the experiment gaze durations on the whole-word were significantly shorter for the hyphen-at-the-major-boundary compounds than for the legally concatenated ones. A similar type of learning was observed in the Dutch experiment; however, it did not result in faster processing of major-boundary hyphenation compounds over concatenated compounds.

The overall pattern of early facilitation offset by later slowing down in processing due to hyphenation is consistent with what Inhoff et al. (2000) found for processing illegally spaced German compound words. In other words, instead of inserting a hyphen at constituent boundaries they added spaces between the constituents in three-constituent compounds (e.g., DatenSchutz Experte). They found shorter gaze durations on illegally spaced than legally unspaced compounds; on the other hand, the final fixation on the word tended to be longer in the spaced than unspaced condition. A similar pattern of results was obtained by Juhasz et al. (2005) for reading normally unspaced English compounds as spaced. First-fixation duration was shorter for spaced than unspaced compounds, but a disruption in processing due to spacing was observed in refixations. Yet, as detailed above, unlike spacing, hyphenation may lead to general processing benefits (see the Finnish results of Bertram et al., 2011; and those of Bertram and Hyönä submitted). This may be due to hyphens signaling that constituents belong to the same unit; on the other hand, spacing cannot accomplish this, which in turn may result in initially interpreting the compound word constituents as belonging to two separate phrases (Staub et al., 2007). 
Bertram et al. (2004) were interested in whether orthographicphonological cues that are more subtle than spaces or hyphens may signal the morphological boundary in long two-constituent Finnish compound words and hence aid in compound word identification. They studied how vowel harmony (vs. disharmony) at the constituent boundary affects the speed of processing twoconstituent compound words in sentence contexts. Vowel harmony refers to a feature in Finnish ${ }^{1}$, where back vowels (a, o, $\mathrm{u}$ ) and front vowels (ä, ö, y) never appear together in word stems or case-inflected words. However, they can co-occur in compound word constituents; for example, the first-constituent may contain front vowels and the second constituent back vowels. Thus, it is also possible to have two vowels of different quality appear adjacent to each other at the morpheme boundary, as in selkäongelma (=back problem; the morpheme boundary is bolded). This is an unambiguous morpheme boundary cue, as the vowels ä and o have to belong to different lexemes. In contrast, the morpheme boundary appears more obscured when two vowels of the same quality stand next to each other at the boundary, as in ryöstöyritys (=robbery attempt; the morpheme boundary is bolded). In the latter case, it is possible to initially misparse the syllable structure of the word, as töy forms an existing syllable (note, however, that the target words never allowed two alternative morphological parses).

In Experiment 1, Bertram et al. (2004) embedded the two types of compound words described above (selkäongelma vs. ryöstöyritys) in sentences and recorded readers' eye movements on these words when silently reading these sentences for comprehension. Vowel quality at the constituent boundary had a significant effect on the speed of word recognition, as indexed by gaze duration on the word; gaze duration was $43 \mathrm{~ms}$ shorter in the vowel disharmony than in the vowel harmony condition. In a follow-up analysis, they compared the vowel harmony effect separately for short (four or five letters) and long (at least six letters) first-constituent compounds (word length was matched). This analysis showed that the effect was doubled in size for long than short first-constituent compounds (23 vs. $49 \mathrm{~ms}$, respectively). The modulation of the effect size is interpreted to be due to visual acuity. The first-fixation on the word landed very close to the morpheme boundary for short first-constituent compounds, while for long first-constituent compounds it was some distance away from the initial fixation. In the former case the entire first-constituent is readily available in foveal vision, whereas in the latter case the morpheme boundary is not exactly at fixation, which then results in the boundary manipulation exerting a bigger effect. The modulation by first-constituent length was further confirmed in Experiment 2, where first-constituent length was systematically varied (three to five vs. seven to nine letters). There was a sizeable vowel harmony effect in gaze duration for long firstconstituent compounds (114 ms), whereas it was non-existent (2 ms) for short first-constituent compounds. Thus, it seems that orthographic-phonological cues help to determine the constituent boundary with long first-constituent compounds, while these cues are ineffective with short first-constituent compounds,

${ }^{1}$ Vowel harmony exists also in Hungarian, distantly related to Finnish, and in some Altaic languages (e.g., Turkish and Uighur). presumably because the boundary is located in the center of the foveal vision when the word is initially fixated.

Two vowels of different quality (front vs. back) at the constituent boundary unavoidably create a bigram trough (Seidenberg, 1987; Rapp, 1992). Thus, the results of Experiment 1 and 2 may not necessarily reflect differences in vowel quality. However, post hoc analyses of Experiment 1 and 2 revealed that the vowel harmony effect was not merely due to differences in the frequency of the bigram spanning the morpheme boundary. Moreover, in Experiment 3 a 60 -ms difference in gaze duration in favor of the disharmony condition over the harmony condition was observed when the critical vowels were not adjacent to each other (i.e., the first-constituent ended with a vowel but the second constituent started with a consonant) and the two vowel harmony conditions were matched for the frequency of the bigram spanning the morpheme boundary. Experiment 3 demonstrates that two vowels of different quality do not need to be adjacent to each other for the effect to emerge. Thus, these data suggest that vowel harmony appears to be a unique defining feature in Finnish for morpheme boundaries, perhaps operating at the phonological level.

In addition to vowel harmony, consonant type at the boundary was also manipulated. In Experiment 3, Bertram et al. (2004) compared two conditions: (a) the initial consonant of the second constituent was such that it cannot appear as the final letter in a lexeme (unambiguous condition), or (b) the consonant was one that can either appear at the end or the beginning of a lexeme (ambiguous condition). Consonant ambiguity produced an effect on gaze duration of similar size $(52 \mathrm{~ms})$ as vowel harmony. The consonant and vowel quality effects appeared independent of each other, as the two factors did not interact with each other. Finally, the analysis of the processing time course of the obtained effects suggested that boundary cue effects peaked at the third fixation made on the word; to a lesser extent they were also apparent during the second and fourth fixation. Thus, the relatively later appearance of the effect is generally inconsistent with the prelexical accounts of morphological decomposition (e.g., Taft, 1979, 1994; Rastle et al., 2004) predicting an early effect.

Interestingly, a recent lexical decision experiment conducted in Dutch (Lemhöfer et al., 2011) found a converging pattern of data to those reviewed above. Lemhöfer et al. observed that lexical decisions to compound words with extremely low-frequency bigrams at the morpheme boundary (e.g., sb in fietsbel) were $26 \mathrm{~ms}$ shorter than those to compounds with a frequent bigram at the boundary (e.g., sp in fietspomp). It should be noted that Inhoff et al. (2000) did not find an effect of uncommon bigrams at the constituent boundary on compound word reading in German. In a follow-up analysis Lemhöfer et al. found, similarly to Bertram et al. (2004), that the boundary cue exerted an effect on the identification of long (10-13 letters) but not of short (6-10 letters) compounds. Curiously, non-native Dutch speakers (German-Dutch bilinguals) did not show the modulation by length. This was taken to suggest that non-native speakers use the decomposition route to identify all compound words, irrespective of length.

In sum, the data summarized above suggest a dynamic interplay between lexical access and morphological parsing during the identification of long two-constituent compounds. Access to the first-constituent is readily achieved when it is short, as the whole 
constituent is within foveal reach during the initial fixation made on the word; thus, morphological parsing cues are of little value and can even be detrimental. In contrast, parsing cues become more valuable in facilitating access to the first-constituent when it is longer and the morpheme boundary resides some distance away from the center of the initial fixation.

\section{WHAT HAPPENS DURING THE FIRST 250 MS OF COMPOUND WORD PROCESSING?}

In this final section I present my view on the topic of the present special issue: what happens within the first $250 \mathrm{~ms}$ of compound word processing. My view is based on the data presented above, the visual acuity principle and the dual-route framework of morphological processing. For the identification of compound words, the dual-route model posits that the whole-word route and the decomposition route operate in parallel and possibly in interaction with each other.

When recognizing compound words that are sufficiently short to fit within the area of the foveal vision, all letters can be identified in parallel, which then enables the activation of the whole-word representation during the initial fixation of the word. Thus, the whole-word route is active early on during processing and dominates the identification of short compound words during those first $250 \mathrm{~ms}$. As the whole-word representation becomes available early on, the initial fixation is often also the only fixation needed to recognize short compound words.

In contrast, simultaneous identification of all letters is impossible with longer compound words; only the letters of the

\section{REFERENCES}

Andrews, S., Miller, B., and Rayner, K. (2004). Eye movements and morphological segmentation of compound words: there is a mouse in the mousetrap. Eur. J. Cogn. Psychol. 16, 285-311.

Bertram, R., and Hyönä, J. (2003). The length of a complex word modifies the role of morphological structure: evidence from eye movements when reading short and long Finnish compounds. J. Mem. Lang. 48, 615-634.

Bertram, R., Kuperman, V., Baayen, R. H., and Hyönä, J. (2011). The hyphen as a segmentation cue in triconstituent compound processing: it's getting better all the time. Scand. J. Psychol. 52, 530-544.

Bertram, R., Pollatsek, A., and Hyönä, J. (2004). Morphological parsing and the use of segmentation cues in reading Finnish compounds. J. Mem. Lang. 51, 325-345.

Calvo, M. G., and Meseguer, E. (2002). Eye movements and processing stages in reading: relative contribution of visual, lexical and contextual factors. Span. J. Psychol. 5, 66-77.

Duñabeitia, J. A., Perea, M., and Carreiras, M. (2007). The role of the frequency of constituents in compound words: evidence from Basque and Spanish. Psychon. Bull. Rev. 14 1171-1176.

Fiorentino, R., and Poeppel, D. (2007). Compound words and structure in the lexicon. Lang. Cogn. Process. 22, 953-1000.

Frisson, S., Niswander-Klement, E., and Pollatsek, A. (2008). The role of semantic transparency in processing of English compound words. $\mathrm{Br}$. J. Psychol. 99, 87-107.

Frost, R. (in press). Towards a universal model of reading. Behav. Brain Sci.

Häikiö, T., Bertram, R., and Hyönä, J. (2011). The development of wholeword representations in compound word processing: evidence from eye fixation patters of elementary school children. Appl. Psycholinguist. 32, 533-551.

Häikiö, T., Bertram, R., Hyönä, J., and Niemi, P. (2009). Development of the letter identity span in reading: evidence from the eye movement moving window paradigm. J. Exp. Child Psychol. 102, 167-181.

Hautala, J., Hyönä, J., and Aro, M. (2011). Dissociating spatial and letter-based word length effects observed in readers' eye movement

Hyönä, J. (2011). "Foveal and parafoveal processing during patterns. Vision Res. 51, 1719-1727.

first-constituent lie in the fovea and are thus recognizable. Consequently, the decomposition route dominates the first $250 \mathrm{~ms}$ of processing. During the initial processing stage, access to the firstconstituent is achieved. A refixation is then needed to identify the remaining letters of the word. The holistic route also becomes fully active during this refixation; yet, the decomposition route is still in operation, as it takes care of the access to the second constituent. The decomposition route is aided by orthographic-phonological cues signaling the morpheme boundary, and with that, the morphological structure of the word. The facilitation in processing due to boundary cues is only achieved when the morpheme boundary is located some distance away from the location of the initial fixation. In other words, when the initial constituent is short, all its letters are clearly visible and boundary cues are not needed to separate its letters from those of the second constituent.

In conclusion, word length strongly affects word identification. Therefore, by widening their scope beyond short words, researchers cannot only generalize their findings to a larger pool of languages, but will also open a treasure trove of valuable new insights regarding early activities in the reading process. In addition, cross-linguistic and multi-language studies are also needed for building word recognition models capable of accounting for data derived from qualitatively different orthographies (see Frost, in press, for further arguments for the need of cross-linguistic studies of word recognition).

\section{ACKNOWLEDGMENTS}

I thank Bernadet Jager for her very helpful comments.

reading," in The Oxford Handbook of Eye Movements, eds S. P. Liversedge, I. Gilchrist, and S. Everling (Oxford: Oxford University Press), 819-838.

Hyönä, J., and Olson, R. K. (1995). Eye fixation patterns among dyslexic and normal readers: effects of word length and word frequency. J. Exp. Psychol. Learn. Mem. Cogn. 21, 1430-1440.

Hyönä, J., and Pollatsek, A. (1998). Reading Finnish compound words: eye fixations are affected by component morphemes. J. Exp. Psychol. Hum. Percept. Perform. 24, 1612-1627.

Inhoff, A. W., Radach, R., and Heller, D. (2000). Complex compounds in German: interword spaces facilitate segmentation but hinder assignment of meaning. J. Mem. Lang. 42, 23-50.

Inhoff, A. W., Starr, M. S., Solomon, M., and Placke, L. (2008). Eye movements during reading of compound words and the influence of lexeme meanings. Mem. Cognit. 36, 675-687.

Juhasz, B. J. (2007). “The influence of semantic transparency on eye movements during English compound word recognition," in Eye
Movements: A Window on Mind and Brain, eds R. P. G. Van Gompel, M. H. Fischer, W. S. Murray, and R. L. Hill (Oxford: Elsevier), 373-389.

Juhasz, B. J. (2008). The processing compound words in English: effects of word length on eye movements during reading. Lang. Cogn. Process. 23, 1057-1088.

Juhasz, B. J., Inhoff, A. W., and Rayner, K. (2005). The role of interwordspaces in the processing of English compound words. Lang. Cogn. Process. 20, 291-316.

Juhasz, B. J., Starr, M. S., Inhoff, A. W., and Placke, L. (2003). The effects of morphology on the processing of compound words: evidence from naming, lexical decision, and eye fixations. Br. J. Psychol. 94, 223-244.

Juhasz, B. J., White, S. J., Liversedge, S. P., and Rayner, K. (2008). Eye movements and the use of parafoveal word length information in reading. J. Exp. Psychol. Hum. Percept. Perform. 34, 1560-1579.

Just, M. A., and Carpenter, P. A. (1980). A theory of reading: from eye fixations to comprehension. Psychol. Rev. 87, 329-354. 
Kliegl, R., Grabner, E., Rolfs, M., and Engbert, R. (2004). Length, frequency, and predictability effects of words on eye movements in reading. Eur. J. Cogn. Psychol. 16, 262-284.

Lemhöfer, K., Koester, D., and Schreuder, R. (2011). When bicycle pump is harder to read than bicycle bell: effects of parsing cues in first and second language compound reading. Psychon. Bull. Rev. 18, 364-370.

McDonald, S. A. (2006). Effects of number-of-letters on eye movements during reading are independent from effects of spatial word length. Vis. Cogn. 13, 89-98.

Pollatsek, A., Bertram, R., and Hyönä, J. (2011). Processing novel and lexicalized Finnish compound words. J. Cogn. Psychol. (Hove) 23, 795-810.

Pollatsek, A., and Hyönä, J. (2005). The role of semantic transparency in the processing of Finnish compound words. Lang. Cogn. Process. 20, 261-290.

Pollatsek, A., and Hyönä, J. (2006). “Processing of morphologically complex words in context: what can be learned from eye movements," in From Inkmarks to Ideas: Current Issues in Lexical Processing, ed. S.
Andrews (Hove: Psychology Press), 275-298.

Pollatsek, A., Hyönä, J., and Bertram, R. (2000). The role of morphological constituents in reading Finnish compound words. J. Exp. Psychol. Hum. Percept. Perform. 26, 820-833.

Rapp, B. C. (1992). The nature of sublexical orthographic organization: the bigram trough hypothesis examined. J. Mem. Lang. 31, 33-53.

Rastle, K., Davis, M. H., and New, B. (2004). The broth in my brother's brothel: morpho-orthographic segmentation in visual word recognition. Psychon. Bull. Rev. 11, 1090-1098.

Rayner, K. (1979). Eye guidance in reading: fixation locations within words. Perception 8, 21-30.

Rayner, K., Well, A. D., and Pollatsek, A. (1980). Asymmetry of the effective visual field in reading. Percept. Psychophys. 27, 537-544.

Rayner, K., Well, A. D., Pollatsek, A., and Bertera, J. H. (1982). The availability of useful information to the right of fixation in reading. Percept. Psychophys. 31, 537-550.

Schotter, E., Angele, B., and Rayner, K. (2012). Parafoveal processing in reading. Atten. Percept. Psychophys. 74, 5-35.
Schreuder, R., and Baayen, R. H. (1995) "Modeling morphological processing," in Morphological Aspects of Language Processing, ed. L. B. Feldman (Hillsdale, NJ: Erlbaum), 131-154.

Seidenberg, M. S. (1987). "Sublexical structures in visual word recognition: access units or orthographic redundancy?" in Attention and Performance XII: The psychology of Reading, ed. M. Coltheart (Hove: Erlbaum), 245-263.

Staub, A., Rayner, K., Pollatsek, A., Hyönä, J., and Majewski, H. (2007) The time course of plausibility effects of eye movements in reading: evidence from noun-noun compounds. J. Exp. Psychol. Learn. Mem. Cogn. 33, 1162-1169.

Taft, M. (1979). Recognition of affixed words and the word frequency effect. Mem. Cognit. 7, 263-272.

Taft, M. (1994). Interactive-activation as a framework for understanding morphological processing. Lang. Cogn. Process. 9, 271-294.

Taft, M., and Forster, K. (1976). Lexical storage and retrieval of polymorphemic and polysyllabic words. J. Verb. Learn. Verb. Behav. 15, 607-620.

Vergara-Martínez, M., Duñabeitia, J. A., Laka, I., and Carreiras, M.
(2009). ERP correlates of inhibitory and facilitative effects of constituent frequency in compound word reading. Brain Res. 1257, 53-64.

Conflict of Interest Statement: The author declares that the research was conducted in the absence of any commercial or financial relationships that could be construed as a potential conflict of interest.

Received: 01 March 2012; accepted: 22 May 2012; published online: 11 June 2012.

Citation: Hyönä J (2012) The role of visual acuity and segmentation cues in compound word identification. Front. Psychology 3:188. doi: 10.3389/fpsyg.2012.00188

This article was submitted to Frontiers in Language Sciences, a specialty of Frontiers in Psychology.

Copyright (C) 2012 Hyönä. This is an open-access article distributed under the terms of the Creative Commons Attribution Non Commercial License, which permits non-commercial use, distribution, and reproduction in other forums, provided the original authors and source are credited. 